\title{
El turismo idiomático en la oferta educativa del Centro de Enseñanza para Extranjeros de la UNAM
}

Luis Miguel Samperio Sánchez Centro de Enseñanza para Extranjeros

\begin{abstract}
Resumen: En este documento se ubica al turismo idiomático como parte de las industrias culturales y de las industrias de la lengua y, se describe la contribución del Centro de Enseñanza para Extranjeros (CEPE) de la UNAM, a esta industrias y al turismo idiomático en México, a través de la actividad académica que realiza el CEPE desde hace casi 100 años. Para ello se presentan las características de su oferta educativa, organizada con la intención de brindar a los estudiantes que aprenden español como lengua extranjera en la UNAM, una experiencia que les permita acceder al conocimiento de las expresiones de la cultura mexicana que a su vez refuerzan su dominio del español, además de los servicios que se les ofrecen a los estudiantes para facilitarles su estadía en la Ciudad de México y el resto del país.
\end{abstract}

EMPRESAS CULTURALES, CREATIVAS Y DEL LENGUAJE

Crovi (2013), señala que la expresión de "industria cultural" fue empleada por primera vez por algunos integrantes de la Escuela de Frankfurt, en particular Adorno y Horkheimer, en la década de los cuarenta del siglo pasado, con el objeto de analizar el cambio que estaba ocurriendo en el terreno social que ocupaba la cultura.

Las industrias culturales integran un buen número de empresas encargadas de la creación, producción, exhibición, distribución y difusión de servicios y bienes culturales, tales como el arte, el entretenimiento, el diseño, la arquitectura, la publicidad, la gastronomía y el turismo; en 2009, la UNESCO las definió como aquellas que combinan la creación, la producción y la comercialización de contenidos creativos, los cuales son intangibles y de naturaleza cultural y que por lo general incorporan a industrias como la imprenta, la editorial, la multimedia, la audiovisual, la discográfica y el cine.

Dentro de las industrias culturales han aparecido otras denominadas "industrias creativas" que, según Toussaint (2013), no las sustituyen, sino que se sirven de ellas en el plano político, económico y de desarrollo social para obtener los mismos beneficios y subsidios. 
No obstante, en el marco de las industrias culturales y las industrias creativas mencionadas, se inscribe un tipo de industria que tiene un referente directo con el objeto de estudio que nos ocupa y es el de la industria del lenguaje, industria lingüística o industria de la lengua cuyo origen es más bien remoto, si tomamos en cuenta la definición que la Asociación de Industrias de la Lengua en el País Vasco (2010) hace de ellas, al considerarlas como el sector de actividad que se encarga de diseñar, producir y comercializar productos y servicios relacionados con el tratamiento de los idiomas. Esta definición permite identificar en ella a un muy amplio y variado espectro de actividades científicas, intelectuales, culturales, educativas y económicas, en el que se incluyen las actividades relacionadas con la enseñanza de idiomas.

Sobre estas industrias se ha reflexionado mucho desde la perspectiva económica, ya que conforman cadenas de valor. Asimismo, desde el ámbito de la lengua, se han revisado las implicaciones en actividades relacionadas con la traducción y la revisión de textos, así como las herramientas de tipo editorial e informático. Particularmente, en la actualidad, encontramos una cantidad importante de aplicaciones en el tratamiento informático, no solo en el software que se utiliza para el procesamiento de textos, sino también para los teléfonos celulares que permiten traducciones simultáneas en muchos idiomas. Quizá las traducciones no sean siempre las mejores, pero a muchos usuarios dejan satisfechos con lo que les ofrecen, dada la relación precio utilidad para resolver un problema de vocabulario en una lengua distinta a la del hablante.

Lo anterior lleva a pensar sobre el papel cada vez más imperativo que está asumiendo la tecnología informática en el aprendizaje y enseñanza de las lenguas extranjeras y en particular de la nuestra. Con el desarrollo de las neurociencias y la inteligencia artificial encontramos más investigaciones que involucran el estudio de los procesos cognitivos de adquisición del lenguaje, que se acompañan de mecanismos informáticos para "facilitar" o evidenciar lo que ocurre en nuestra mente cuando hablamos y la forma en la que estructuramos nuestro pensamiento para hablar, tal como lo destaca Vidal (1987), es un tema que vincula muy explícitamente a la industria de las tecnologías de la información, en la que interviene la lingüística, la inteligencia artificial, la ingeniería de cómputo, el desarrollo de software y de interfaces biotecnológicas, entre otras.

Durante el tiempo que va de este siglo se han incrementado las comunicaciones que se refieren a la llamada "singularidad tecnológica", definida de una manera muy elemental, como el momento en el que el proceso evolutivo de los seres humanos será inducido por las tecnologías emergentes, permitiéndole alcanzar a éstos, de acuerdo con Cortina (2015), una superinteligencia, una superlongevidad y un superbienestar desconocidos por la humanidad hasta el día de hoy. Especulando un poco, la inteligencia 
artificial superará a la inteligencia humana y esto tendrá un impacto muy significativo en el aprendizaje y la enseñanza de las lenguas extranjeras, ya que muy probablemente no será necesario acudir a algún centro o lugar para aprender una lengua sino que, a la manera de las clásicas novelas "ciber punk", podremos instalar en nuestro encéfalo una interface biotecnológica, que nos permitirá conectar una fuente de información en el que vaya todo lo necesario para, instantáneamente, comunicarnos en cualquier lengua extranjera distinta a la materna.

TURISMO IDIOMÁTICO

El turismo se puede ubicar dentro del rubro de las industrias culturales y la Organización Mundial del Turismo (OMT) lo define como, las actividades que realizan las personas durante sus viajes y estancias en lugares distintos a su entorno habitual por un período de tiempo consecutivo inferior a un año, con fines de ocio, negocios u otros.

Podemos hablar entonces del turismo cultural como la actividad que considera la estancia, el tiempo y la finalidad de un paseante relacionados no solo con el patrimonio histórico y artístico del lugar que visita, sino que abarca otras áreas materiales e inmateriales del mismo, tales como la artesanía, la gastronomía, la música y el idioma, sobre este último es que se desarrolla el turismo lingüístico o idiomático.

La OMT reconoce al turismo idiomático desde 1991 y lo define como "las actividades que realizan las personas durante sus viajes y estancias en lugares distintos al de su entorno natural por un período de tiempo consecutivo inferior a un año con el fin de hacer una inmersión lingüística en un idioma distinto al de su entorno natural".

Al día de hoy existe una alta y constante demanda de destinos turísticos por parte de aquellos que desean aprender otro idioma. El caso de nuestra lengua es muy relevante, según datos del Instituto Cervantes (2018) hay más de 21 millones de personas en el mundo estudiando español como lengua extranjera. Por eso, en el CEPE se continúa con la enseñanza del español como lengua extranjera, en el marco de la cultura mexicana como se ha venido haciendo desde hace más de 97 años. 


\section{La oferta educativa del Centro de Enseñanza para Extranjeros de la UNAM}

Partiendo de la definición del turismo idiomático planteada por la OMT, en los siguientes párrafos se presenta la contribución práctica de la UNAM al turismo idiomático a través del Centro de Enseñanza para Extranjeros (CEPE), como una expresión de las industrias culturales y también a las de la lengua, ya que en términos editoriales, contamos con una cadena de valor que abarca el diseño, la producción y comercialización del material didáctico en formato de libros que emplean sus estudiantes.

EI CEPE, como ya se dijo, cuenta con una dilatada experiencia de casi 100 años en la enseñanza del español como lengua extranjera, en el marco de la cultura mexicana expresada en tres disciplinas: arte, historia y literatura; lo que ha contribuido a través de la enseñanza, la formación de profesores y la certificación del dominio de nuestra lengua a su enriquecimiento $y$, de manera paulatina y a veces no intencional, al crecimiento del turismo idiomático en nuestro país.

Además tiene una oferta de cursos de español y cultura mexicana para no hispanohablantes, que se complementa con un programa de paseos culturales y otro de actividades lúdicas diseñados especialmente para fortalecer el aprendizaje de nuestra lengua. La certificación está integrada al programa de español y cultura, ya que al término de sus cursos los estudiantes presentan el examen SIELE en su modalidad S1, que mide comprensión de lectura y auditiva, para la evaluación del nivel alcanzado en el dominio del español como producto de nuestros cursos. Lo anterior se representa en la Figura 1.

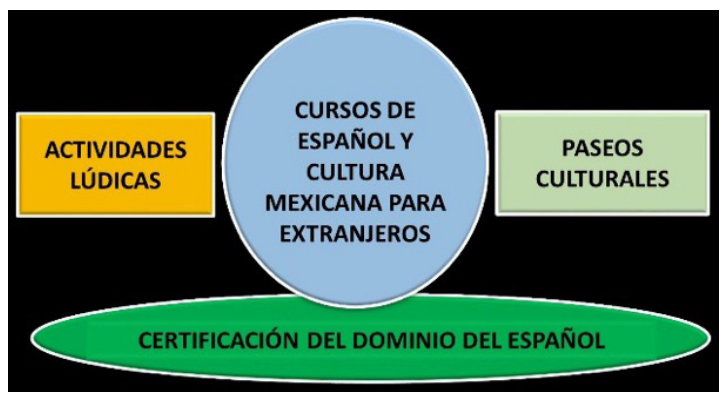

Figura 1. Modelo de cursos de español y cultura mexicana para extranjeros

Para operar este esquema, la oferta de cursos de español y cultura mexicana para extranjeros se organiza en nueve niveles, desde un nivel de iniciación hasta el superior. Todos los cursos se ofrecen en la modalidad presencial y de manera intensiva en seis periodos al año. Tienen una duración de 126 horas, que se imparten a lo largo de seis semanas, lo que significa que durante este tiempo 
los estudiantes asisten en promedio cuatro horas y media diarias a sus cursos de español y cultura mexicana. Los cursos en los primeros cinco niveles tienen 90 horas de español, además de 18 de práctica de español y 18 de cultura. Las horas destinadas a esta última se incrementan significativamente conforme avanzan en el dominio de la lengua, lo que les permite acceder a conocimientos de la cultura mexicana más complejos. La expresión de la distribución horaria se puede observar en la siguiente figura.

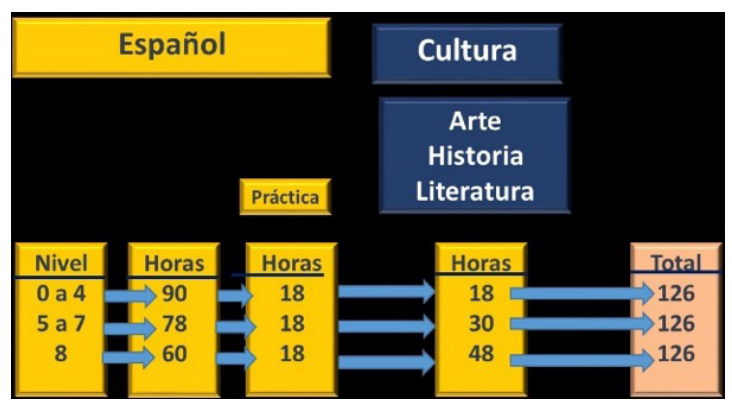

Figura 2. Cursos de español y cultura mexicana para extranjeros

Desde que el CEPE fue fundado como Escuela de Verano en 1921, ha tenido como parte de sus actividades académicas, un programa de visitas guiadas a sitios de interés histórico y artístico en la Ciudad de México y lo que en esa época se consideraban "los alrededores de México". También se organizaban paseos a las zonas arqueológicas más cercanas a la Ciudad.

El objetivo de estas visitas era que los estudiantes extranjeros y mexicanos, además de conocer todos esos lugares, profundizaran en sus conocimientos sobre la cultura mexicana. A partir de entonces, las visitas guiadas han formado parte fundamental del programa de estudios, por lo que se han ofrecido de manera ininterrumpida hasta el presente año.

Actualmente los estudiantes extranjeros del CEPE, como parte de sus cursos, realizan un paseo cultural guiado por un docente, cuyo objetivo es fomentar el conocimiento y el aprecio por la cultura mexicana, además de apoyar el aprendizaje del español de acuerdo con el nivel de su curso. Estas visitas son definidas de conformidad con cinco ejes temáticos que organizan los contenidos de los cursos de cultura y que se muestran en la Figura 3. 

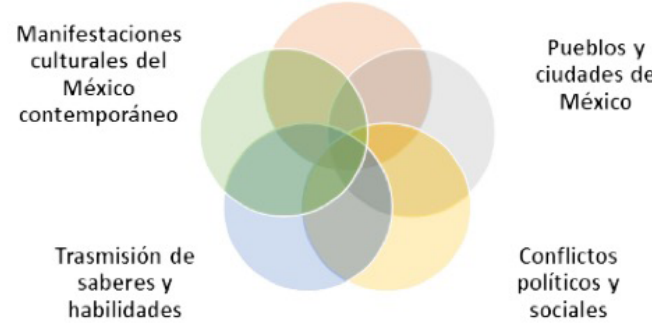

Figura 3. Ejes temáticos de los cursos de cultura mexicana

Para fortalecer las acciones educativas del modelo de enseñanza-aprendizaje del español, se han desarrollado materiales educativos que son resultado de años de experiencia y vocación docente. Desde hace casi 30 años, se ha contribuido a la industria de la lengua, ya que, como se dijo líneas arriba, se han diseñado, producido, publicado y comercializado cuatro series de libros: "Pido la palabra" (1988), "Estoy listo" (1994), "Así hablamos. Español como lengua extranjera" (2007), y“Dicho y hecho. Español como lengua extranjera” (2014) que es la serie que actualmente se utiliza en los cursos.

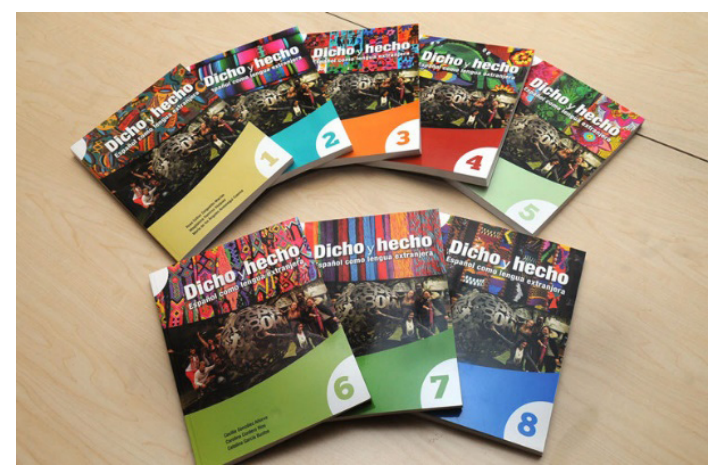

Figura 4. Serie de libros "Dicho y hecho. Español como lengua extranjera"

Como parte de los servicios de apoyo a sus estudiantes, en el CEPE también se ofrece orientación personalizada para que puedan ubicarse y moverse no sólo dentro del campus universitario, sino en la compleja zona metropolitana 
de la Ciudad de México. Además, se cuenta con una atención individual y la posibilidad de utilizar un sistema informático para conseguir hospedaje en lugares cercanos al campus, ya que desde hace poco más de medio siglo hemos organizado una amplia red de prestadores de servicio, a través de un sistema de alojamiento, que ofrece opciones de hospedaje de acuerdo con diversas condiciones de espacio físico, ubicación, ambiente, precio y seguridad. En el sistema mencionado, los usuarios tienen acceso a diversas opciones para que elijan y decidan libremente la que más les agrade o convenga a sus necesidades. Se busca que las casas y departamentos, seleccionados por personal del CEPE, cumplan con los requisitos mínimos de seguridad, limpieza, higiene, ambiente familiar y medios de transporte.

Otro servicio educativo con el que se busca impulsar el turismo idiomático y atender necesidades específicas que no se puedan cubrir con los cursos regulares, es la oferta de cursos a la medida, dirigidos a grupos de 10 a 15 estudiantes, mayores de 18 años, sin distingo de raza, género, orientación sexual, nacionalidad, lengua o religión. Esta oferta no solo considera los requerimientos de aprendizaje del español y tiempo que puedan dedicarle los estudiantes, que puede ser de una hasta diez semanas, sino que incorpora otros aspectos como el hospedaje, alimentación, transportación y seguro contra accidentes, que hacen más agradable y segura la experiencia de aprender español en México. En la siguiente figura se mencionan las principales características de la oferta.

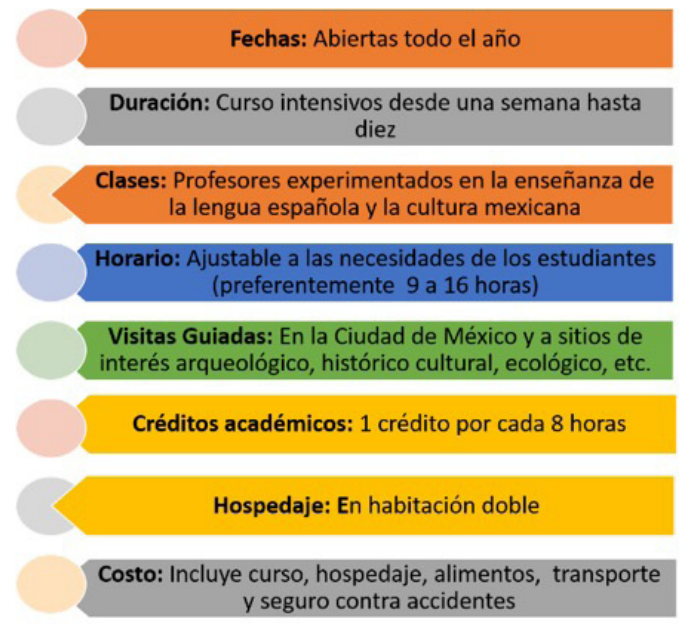

Figura 5. Características de la oferta de cursos a la medida 
CONCLUSIONES.

La intención de la oferta educativa del CEPE de cursos para no hispanohablantes es hacer que quienes estudien español como lengua extranjera en la UNAM, además de beneficiarse de la experiencia de casi un siglo de enseñar español, lo hagan en un entorno que resulte en una vivencia que quieran repetir y en su momento sean promotores de lo que hacemos en México y no solo en la enseñanza del español, sino que se vayan enriquecidos con los elementos de la extensa y variada cultura mexicana, además de la experiencia intercultural de haber convivido con estudiantes de más de 80 países de todos los continentes.

En la medida que cada vez más estudiantes asistan a cualquiera de sus cursos, se seguirá contribuyendo, como seguro lo hacen otras instituciones nacionales, al turismo idiomático en México, donde esta actividad económica tiene una regulación muy incipiente para su promoción e incentivación. A través de la serie de libros de "Dicho y hecho" el CEPE también contribuye a promover el turismo idiomático, ya que en ellos se abordan temas que hacen referencia, entre otros, a sitios arqueológicos o de interés histórico de nuestro país, así como museos, costumbres y tradiciones y los lugares donde se llevan a cabo, invitando con esto a los estudiantes a que conozcan México.

En el CEPE se tiene mucha claridad en que, al enseñar español como lengua extranjera, se contribuye al enriquecimiento de la lengua española y al de los diversos campos que se dedican a las expresiones de la multi e interculturalidad. Además de hacerlo con los mecanismos, técnicas, instrumentos y recursos metodológicos, pedagógicos y didácticos que se emplean en la docencia del español y de las lenguas extranjeras.

EI CEPE como parte de una institución pública de educación superior, no tiene como objetivo crear cadenas de valor que generen ganancias para ningún accionista, no obstante, la masa crítica que se genera como parte del quehacer educativo de sus cuerpos docentes, ante la necesidad de contar con herramientas que faciliten la enseñanza y promuevan el aprendizaje del español, lo han llevado a que el diseño, edición, producción y venta de sus materiales didácticos en formato de libros, sean una cadena de valor que, de acuerdo con las definiciones arriba presentadas, son características de una empresa que se ubica en el terreno de las industrias culturales y de la lengua. Lo anterior debe mantenernos en la reflexión, al menos en las universidades públicas, sobre el papel empresarial que asumen estas instituciones cuando por necesidad, y sobre todo por contribuir al conocimiento, generan cadenas de valor.

Por último, dejo un atisbo de preocupación sobre las consecuencias que en el futuro cercano tendrá el desarrollo tecnológico aplicado a las neurociencias y 
la inteligencia artificial, para la enseñanza y el aprendizaje de las lenguas en el mundo y en particular del español como lengua extranjera, pues como ya se dijo, la existencia en el mercado de traductores que son sucedáneos de lo que una persona necesita para comunicarse en nuestra lengua, cada vez son más socorridos en su uso. Tampoco hay que olvidar que empresas como Microsoft y otras tantas, están haciendo inversiones multimillonarias que ninguna universidad o gobierno podrán hacer en el terreno de las lenguas, para que los futuros usuarios puedan realizar traducciones simultáneas en casi cualquier lengua.

En fin, aún tenemos mucho que considerar para que integremos la tecnología en los procesos de enseñanza y aprendizaje del español, por lo que debemos apoyar y fortalecer los mecanismos que favorezcan que la experiencia de aprender nuestra lengua por un no hispanohablante sea en presencia de personas con todos los sentidos bien puestos y en condiciones que faciliten esos procesos, amén de que resulte una experiencia agradable. 


\section{REFERENCIAS}

Asociación de Industrias de LA lengua en el país Vasco (s.f.) Industrias de la lengua Recuperado el 22 de febrero de 2019 de http://www.langune.com/presentacion/ industrias-de-la-lengua

CEPE (2018). Recuperado el 5 de marzo de 2019 de http://cepe.unam.mx

Cortina, A. (2015). Transhumanismo y singularidad tecnológica: superinteligencia, superlongevidad y superbienestar. Recuperado el 20 de febrero de 2019 de http://www.fragmenta.cat/ponencia-de-albert-cortina_420424.pdf

CROvI, D. (2013). Industrias culturales en México. Estrategias y políticas gubernamentales. En Industrias culturales. Reflexiones para actualizar el debate. UNAM, México.

Instituto Cervantes (2018). 577 millones de personas hablan español, el 7,6 $\%$ de la población mundial. Recuperado el 15 de febrero de 2019 de https:// www.cervantes.es/sobre_instituto_cervantes/prensa/2018/noticias/np_ presentacion-anuario.htm

Organización Mundial del Turismo (2018) ¿Por qué el Turismo? Recuperado el 15 de febrero de 2019 de http://www2.unwto.org/es/content/por-que-el-turismo

Toussaint, F. (2103). Evolución de las industrias de la cultura (1980-2010.) En Industrias culturales. Reflexiones para actualizar el debate. UNAM, México.

UNESCO (2017) Políticas para la creatividad. Guía para el desarrollo de las industrias culturales y creativas. Recuperado el 20 de febrero de 2019 de http://www.unesco.org/new/es/culture/themes/cultural-diversity/diversityof-\%09cultural-expressions/tools/policy-guide/como-usar-esta-guia/sobredefiniciones-\%09que-se-entiende-por-industrias-culturales-y-creativas/

VIDAL, J. (1987) Industrias de la lengua. Recuperado el 17 de marzo de 2019 de https://elpais.com/ 